\section{OP0284 MUC5B PROMOTER VARIANT RS35705950 IS A RISK FACTOR FOR RHEUMATOID ARTHRITIS - INTERSTITIAL LUNG DISEASE}

P.-A. Juge ${ }^{1 *}$, J.S. Lee ${ }^{2}$, E. Ebstein ${ }^{1}$, H. Furukawa ${ }^{3}$, E. Dobrinskikh ${ }^{2}$, S. Gazal ${ }^{4}$ C. Kannengiesser ${ }^{5}$, S. Ottaviani ${ }^{1}$, N. Tsuchiya ${ }^{3}$, S. Oka ${ }^{3}$, S. Tohma ${ }^{3}$, J. RojasSerrano ${ }^{6}$, M.-I. Gonzalez-Perez ${ }^{6}$, M. Mejia ${ }^{6}$, I. Buendia-Roldan ${ }^{6}$, R. FalfanValencia $^{6}$, E. Manali ${ }^{7}$, S.A. Papiris ${ }^{7}$, T. Karageorgas ${ }^{8}$, D. Boumpas ${ }^{8}$, K. Antoniou ${ }^{9}$, C. Van Moorsel ${ }^{10}$, J. van der Vis ${ }^{10}$, Y. de Man ${ }^{10}$, J. Grutters ${ }^{10}$, Y. Wang ${ }^{11}$, R. Borie ${ }^{12}$, L. Wemeau-Stervinou ${ }^{13}$, B. Wallaert ${ }^{13}$, R.-M. Flipo ${ }^{14}$, H. Nunes ${ }^{15}$ D. Valeyre ${ }^{15}$, N. Saidenberg ${ }^{16}$, S. Marchand-Adam ${ }^{17}$, K. Deane ${ }^{2}$, A. Walts ${ }^{2}$, T. Fingerlin ${ }^{18}$, E. Matteson ${ }^{19}$, T. Niewold ${ }^{19}$, D. Assayag ${ }^{20}$, A. Gross ${ }^{21}$, P. Wolters ${ }^{21}$, M. Schwarz ${ }^{2}$, M. Holers' ${ }^{2}$, J. Solomon ${ }^{22}$, T. Doyle ${ }^{23}$, M.-P. Debray ${ }^{24}$, C. Boileau ${ }^{5}$, B. Crestani ${ }^{12}$, D. Schwartz ${ }^{2}$, P. Dieudé ${ }^{1}{ }^{1}$ Rheumatology, HOPITAL BICHAT, Paris, France; ${ }^{2}$ Medicine, University of Colorado School of Medicine, Aurora, USA ${ }^{3}$ Molecular and Genetic Epidemiology Laboratory, Faculty of Medecine, University of Tsukuba, Tsukuba, Japan; ${ }^{4}$ Department of Epidemiology, Harvard T.H. Chan School of Public Health, Boston, USA; ${ }^{5}$ Genetics, HOPITAL BICHAT, Paris, France; ${ }^{6}$ Interstitial Lung Disease and Rheumatology Unit, Instituto Nacional de Enfermedades Respiratorias, Ismael Cosío Villegas, Mexico City, Mexico; ${ }^{7}$ 2nd Pulmonary Medicine Department, ${ }^{8}$ Rheumatology and Clinical Immunology Unit, 4th Department of Internal Medicine, University Hospital of Athens 'Attikon', National and Kapodistrian University of Athens, Athens: ${ }^{9}$ PS Department of Respiratory Medicine and Laboratory of Molecular and Cellular Pneumonology, Faculty of Medicine, University of Crete, Heraklion, Greece; ${ }^{10}$ St Antonius ILD center of excellence, Nieuwegein, Netherlands; ${ }^{11}$ Department of Medical Genetics, Nanjing University School of Medicine, Nanjing, China; ${ }^{12}$ Pneumologie A, HOPITAL BICHAT, Paris; ${ }^{13}$ Service de Pneumologie; ${ }^{14}$ Service de Rhumatologie, CHRU de Lille, Lille; ${ }^{15}$ Service de Pneumologie; ${ }^{16}$ Service de Rhumatologie, Hôpital Avicenne, Bobigny; ${ }^{17}$ Service de Pneumologie, CHRU de Tours, Tours, France; ${ }^{18}$ National Jewish Health, Denver, ${ }^{19}$ Pulmonary, Mayo Clinic, Rochester, USA; ${ }^{20}$ Medicine, McGill University, Montreal, Canada; ${ }^{21}$ Medicine, UCSF, San Francisco; ${ }^{22}$ Medicine, National Jewish Health, Denver, ${ }^{23}$ Medicine, Brigham and Women's Hospital, Boston, USA $;{ }^{24}$ Radiologie, Hôpital Bichat, Paris, France

Background: Rheumatoid arthritis-associated interstitial lung disease (RA-ILD) and idiopathic pulmonary fibrosis (IPF) share phenotypic similarities. The gain-offunction MUC5B promoter variant rs35705950 is the strongest risk factor for development of IPF

Objectives: We hypothesised that rs35705950 would also contribute to the risk of ILD in RA patients.

Methods: Using a French discovery population and multi-ethnic validation populations from 6 different countries, we tested the association of the MUC5B promoter variant in RA-ILD ( $n=620)$, RA without ILD ( $n=614)$, and unaffected controls $(n=5448)$.
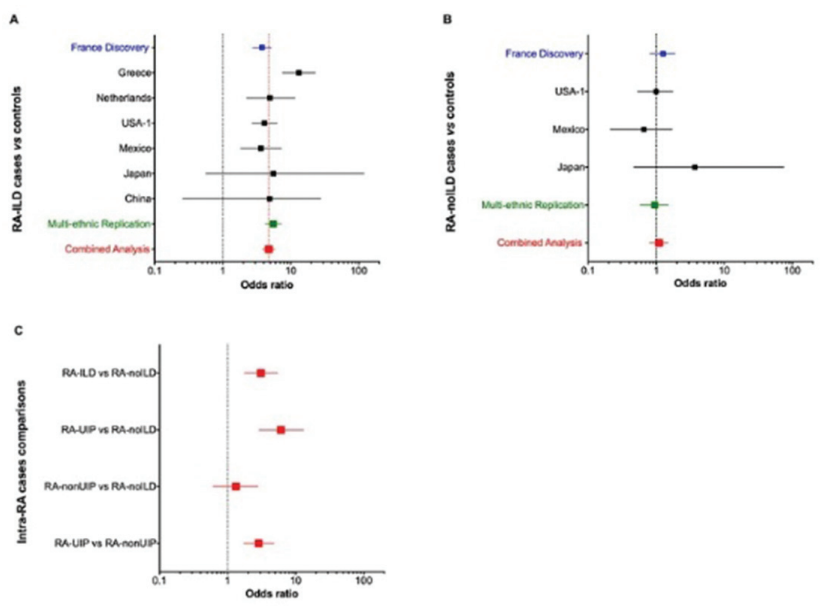

Results: The discovery population revealed an association of the MUC5B promoter variant with RA-ILD when compared to unaffected controls $\left(\mathrm{OR}_{\mathrm{adi}}=3.8\right.$ $95 \% \mathrm{Cl}: 2.8$ to $5.2 ; p=9.7 \times 10-1^{7}$ ) (figure $1 A$ ). Similar to the discovery cohort, the MUC5B promoter variant was significantly over-represented among the cases of RA-ILD in the multi-ethnic study cohorts when compared to unaffected controls $\left(\mathrm{OR}_{a d}=5.595 \% \mathrm{Cl}: 4.2\right.$ to $\left.7.2 ; \mathrm{p}=4.7 \times 10-3^{5}\right)$ (figure $\left.1 \mathrm{~A}\right)$, and when the discovery population and the multi-ethnic cohorts were combined $\left(\mathrm{OR}_{\text {combined }}=4.795 \% \mathrm{Cl}\right.$ : 3.9 to $5.8 ; p=1.3 \times 10-4^{9}$ ) (figure $1 A$ ). Additionally, the MUC5B promoter variant was found to increase the risk of ILD among patients with RA ( $\mathrm{OR}_{\text {combined }}=3.1$ $95 \% \mathrm{Cl}: 1.8$ to $5.4 ; p=7.4 \times 10-5)$, however, no statistical association with the MUC5B promoter variant was observed for RA without ILD (figure 1B). The association of the MUC5B promoter variant with RA-ILD increased significantly when restricted to the usual interstitial pneumonia (UIP) by high-resolution computed tomography $\left(\mathrm{OR}_{\text {combineo }}=6.195 \% \mathrm{Cl}: 2.9\right.$ to $\left.13.1 ; \mathrm{p}=2.5 \times 10-6\right)$ (figure $\left.1 \mathrm{C}\right)$. Immunohistochemical and in-situ hybridization analysis of RA-ILD lung tissue demonstrated expression of MUC5B by type 2 alveolar epithelial cells undergoing endoplasmic reticulum stress.

Conclusions: Our findings demonstrate that $M U C 5 B$ promoter variant rs35705950 is a risk factor for RA-ILD specifically associated with radiologic evidence of UIP.

Disclosure of Interest: None declared

DOI: 10.1136/annrheumdis-2018-eular.4727

\section{OP0285 IDENTIFICATION OF RARE CODING VARIANTS IN IL-1- RELATED PATHWAYS IN PATIENTS WITH ADULT- ONSET STILL'S DISEASE}

G Cavalli $^{1}, 2$, R. van Deuren ${ }^{2}$, P. Arts ${ }^{2}$, M. Steehouwer ${ }^{2}$, C. Gilissen ${ }^{2}$, P. Sfriso ${ }^{3}$, P. Galozzi ${ }^{3}$, S. Colafrancesco ${ }^{4}$, R. Priori ${ }^{4}$, L. Cantarini ${ }^{5}$, O.M. Lucherini ${ }^{5}$, S. Rodolfi ${ }^{1}$, G. De Luca ${ }^{1}$, E. Baldissera ${ }^{1}$, F. van de Veerdonk ${ }^{2}$, L. Dagna ${ }^{1}$, A. Hoischen ${ }^{2}$, C.A. Dinarello ${ }^{2,6} .{ }^{1}$ Vita-Salute San Raffaele University, Milan, Italy; ${ }^{2}$ Radboud University Medical Center, Nijmegen, Netherlands; ${ }^{3}$ University of Padua, Padua; ${ }^{4}$ Sapienza University of Rome, Rome; ${ }^{5}$ University of Siena, Siena, Italy;

${ }^{6}$ University of Colorado Denver, Aurora, CO, USA

Background: Adult-onset Still's disease (AOSD) is a rare autoinflammatory disease characterised by fever, arthritis, and multi-organ involvement. Inflammation in AOSD is mediated by interleukin (IL) $-1 \beta$, as confirmed by the dramatic clinical efficacy of selective blockers of this cytokine. The genetic predisposition to this rampant IL-1-driven inflammation remains nevertheless elusive. Previous studies failed to identify associations between polymorphisms in the genes encoding IL-1 and AOSD, thus pointing at more complex genetic mechanisms. This 'missing heritability' cannot be adequately investigated with traditional techniques for genetic partitioning, such as GWAS, which only assess common variants and polymorphisms. Studies focusing on highly penetrant rare variants or different types of mutations (i.e. small copy-number variations; insertions/deletions) are warranted.

Objectives: We hypothesised that genetically determined changes in IL-1related pathways resulting in excessive IL-1 $\beta$ activity lead to the development of autoinflammation in AOSD. Scope of this study was to unravel the combined mutational variation of a network of IL-1-related receptors, pathways, counter-regulators, and cellular processes possibly involved in the pathogenesis of AOSD and IL-1-mediated inflammation in general.

Methods: We collected clinical, demographic, and genetic data from a large cohort of 76 AOSD patients and developed an innovative platform based on molecular inversion probes (MIP) technology for performing highly multiplexed targeted-resequencing. This allows efficient sequencing of the coding sequence of 48 genes related to the IL-1-pathway, and allows studying rare and common variants in one assay. We have also screened 500 healthy controls, and 1000 s of samples with other disorders using the same assay.

Results: We identified rare and unique (i.e. private variants) in the IL1 pathway in several individuals with AOSD. Whether any these are involved in a strong predisposition to AOSD is currently followed-up. Rare genetic variants have been identified in six IL-1-pathway 'clusters':

1. Deregulated activation of the inflammasome and release of IL-1 $\beta$ and IL18.

2. IL-1 family receptors and intracellular signalling mediators.

3. Other pro-inflammatory cytokines and receptors.

4. Regulatory molecules, including IL-1Ra or IL-37.

5. Cellular processes regulating production of $\mathrm{IL}-1$ and $\mathrm{IL}-18$ (i.e. autophagy).

6. Production of ROS, which function as markers of cellular damage and trigger inflammation.

Conclusions: Unravelling the genetic bases of inflammation in AOSD deepens our understanding of the human innate immunome. Of note, this study platform may serve for the genetic analysis of other IL-1-mediated conditions, including gout and other autoinflammatory diseases, whose genetic predisposition remains elusive. Equally important, the identification of pathways amenable to targeting with small molecules or biologics may translate into remarkable clinical implications.

Disclosure of Interest: None declared

DOI: 10.1136/annrheumdis-2018-eular.3454 